\title{
ENTHOUSIASME ET SORCELLERIE CHEZ HENRY MORE ET JOSEPH GLANVILL : LES LIMITES DE LA CRITIQUE DE L'ENTHOUSIASME
}

\author{
Claire Crignon de Oliveira
}

Presses Universitaires de France | Revue de métaphysique et de morale

\author{
$2008 / 3-n^{\circ} 59$ \\ pages 323 à 335
}

ISSN 0035-1571

Article disponible en ligne à l'adresse:

http://www.cairn.info/revue-de-metaphysique-et-de-morale-2008-3-page-323.htm

Pour citer cet article :

Crignon de Oliveira Claire, «Enthousiasme et sorcellerie chez Henry More et Joseph Glanvill : les limites de la critique de l'enthousiasme »,

Revue de métaphysique et de morale, 2008/3 n59, p. 323-335. DOI : 10.3917/rmm.083.0323

Distribution électronique Cairn.info pour Presses Universitaires de France.

(C) Presses Universitaires de France. Tous droits réservés pour tous pays.

La reproduction ou représentation de cet article, notamment par photocopie, n'est autorisée que dans les limites des conditions générales d'utilisation du site ou, le cas échéant, des conditions générales de la licence souscrite par votre établissement. Toute autre reproduction ou représentation, en tout ou partie, sous quelque forme et de quelque manière que ce soit, est interdite sauf accord préalable et écrit de l'éditeur, en dehors des cas prévus par la législation en vigueur en France. II est précisé que son stockage dans une base de données est également interdit. 


\title{
Enthousiasme et sorcellerie chez Henry More et Joseph Glanvill : les limites de la critique de l'enthousiasme
}

\begin{abstract}
RÉSUMÉ. - Henry More (1614-1687) et Joseph Glanvill (1636-1680), connus pour avoir imposé le principe d'une explication naturelle et rationnelle de l'enthousiasme, refusent d'étendre cette explication au phénomène de la sorcellerie. Au lieu d'interpréter cette prise de position paradoxale comme le signe d'une résistance de l'obscurantisme au progrès des Lumières, cet article montre comment la défense de la croyance en la sorcellerie permet d'éclairer le sens et les limites de la critique philosophique de l'enthousiasme telle qu'elle est menée par ces deux grandes figures du platonisme de Cambridge.
\end{abstract}

ABSTRACT. - Henry More (1614-1687) and Joseph Glanvill (1636-1680), known for their rational and natural explanation of enthusiasm, refuse to apply this explanation to the phenomenon of witchcraft. Instead of interpreting this paradoxical stand as a sign of resistance from obscurantism to the growth of reason, this paper shows that the defence of the belief in witchcraft helps to understand the meaning and limits of the philosophical criticism of enthusiasm as it is dealt with by these two leading figures of Cambridge platonism.

On peut difficilement évoquer la figure de l'enthousiaste en Angleterre au $\mathrm{XVII}^{\mathrm{e}}$ siècle sans rencontrer celle du sorcier ou de la sorcière. Les sorcières, comme les enthousiastes, sont créditées d'un certain nombre de dons extraordinaires : la divination, la capacité à s'exprimer en une langue étrangère sans l'avoir apprise, celle de communiquer avec des esprits d'un autre monde, de préserver la santé ou bien au contraire de l'altérer, etc. '. Mais la question n'est pas tant de savoir jusqu'à quel point il est possible de comparer deux types de comportements - qui peuvent tous deux être perçus comme une forme de menace politique et religieuse - que de se demander si une même hypothèse explicative peut permettre de comprendre l'enthousiasme et la sorcellerie.

1. Reginald ScOT, The Discoverie of Witchraft (1584), reprint of the first edition published in 1584, ed. Brinsley Nicholson, London, 1973, chap. XI, p. 45. 
Le débat concernant la sorcellerie se présente très vite sous la forme d'une polémique. Celle-ci oppose les partisans de l'explication médicale qui - comme Jean Wier ${ }^{2}$ en Suisse ou Reginald Scot en Écosse - interprètent les prétendus dons des sorcières comme les symptômes d'un dérèglement pathologique de l'humeur mélancolique et plaident en faveur d'un traitement thérapeutique en lieu et place de la persécution qui sévit à l'époque, aux partisans de l'explication démonologique - Jean Bodin ${ }^{3}$ en France ou bien le roi Jacques I ${ }^{\mathrm{er}} 4$ en Angleterre - persuadés quant à eux de la réalité des dons surnaturels qui sont attribués aux sorcières et de leur commerce avec le diable. Il y a là, selon eux, une véritable menace politique et religieuse à laquelle il convient de répondre par la thèse de la culpabilité des sorcières et non par l'explication médicale qui revient à transformer ces dernières en victimes.

Or cette question (les sorcières sont-elles coupables d'un commerce avec le diable ou bien sont-elles tout simplement victimes d'un dérèglement pathologique de leur humeur) se pose aussi de manière cruciale pour les enthousiastes, en particulier sous sa forme religieuse : ceux qui prétendent être en communication directe avec Dieu par le biais de l'inspiration, de la prophétie, ceux qui se séparent de l'orthodoxie religieuse pour apporter une nouvelle révélation, doivent-ils être considérés eux aussi comme les victimes d'un désordre mélancolique et d'une perturbation de leur faculté d'imaginer ou bien sont-ils, comme les sorcières, des agents du diable, réellement investis de pouvoirs surnaturels au moyen desquels ils agissent contre les intérêts de la vraie religion?

Henry More (1614-1687) et Joseph Glanvill (1636-1680) sont tous deux connus pour avoir proposé une explication rationnelle du comportement des enthousiastes religieux, qu'il s'agisse des puritains se réclamant de la réforme calviniste ou des membres des sectes qui se sont multipliées pendant la guerre civile. Partisans d'une pratique de la religion fondée sur un usage modéré et sobre de la raison, on s'attend à ce qu'ils admettent au sujet de la sorcellerie le même type d'explication naturaliste qu'au sujet des enthousiastes ${ }^{5}$. Et cela d'autant plus qu'à l'époque où ils écrivent (dans les années 1650-1670), on admet beaucoup plus facilement l'explication médicale au sujet des sorcières

2. Jean WIER, Cinq Livres de l'imposture et tromperie des diables et sorcelleries, Paris, Jacques Puys, 1569 (rééd. Hachette, 1975). 1979.

3. Jean Bodin, De la Démonomanie des Sorciers (1580), éd. J. du Puys, Gutemberg Reprints,

4. JACQUES $\mathrm{I}^{\mathrm{er}}$ (James I), Daemonologie, in forme of a Dialogue, divided into three bookes, Edinburgh, 1597, facsimile ed., Amsterdam, Da Capo Press, 1969.

5. Voir à ce sujet les travaux de Michel HeYd et en particulier "Be Sober and Reasonable". The Critique of Enthusiasm in the Seventeenth and Early Eighteenth Centuries, Leiden, E. J. Brill, 1995. 
qu'au sujet des enthousiastes. En effet, l'enthousiasme est encore, après la guerre civile et pendant plusieurs décennies, considéré comme l'une des principales causes des désordres de la guerre civile et beaucoup plaident en faveur d'une explication démonologique ou politique qui leur semble plus efficace pour contrer ce danger que l'explication médicale. Au contraire, la croyance en la réalité des dons attribués aux sorcières tend à diminuer pendant la même période. Dans les années 1650, plusieurs auteurs dénoncent les procès en sorcellerie et les exécutions de sorcières : c'est le cas par exemple de Robert Filmer dans l'Advertisment to the Jurymen of England (1656).

Et pourtant, loin d'étendre au cas des sorcières le principe d'une explication médicale qu'ils défendent au sujet des enthousiastes, More et Glanvill œuvrent (militent même) en faveur de la croyance en la réalité des dons surnaturels attribués aux sorcières. C'est donc sous la forme d'un paradoxe, au moins apparent, que la question du rapport entre enthousiasme et sorcellerie se pose chez eux. Ces auteurs qui plaident en faveur d'une conciliation possible de la foi et de la raison apparaissent pourtant comme responsables de la persistance, au XVII ${ }^{\text {e }}$ siècle, des préjugés les plus tenaces au sujet de la sorcellerie ${ }^{6}$.

Peut-on pour autant se contenter d'opposer ainsi le rationalisme de More et de Glanvill au sujet de l'enthousiasme à leur obscurantisme ou à leur aveuglement concernant la sorcellerie ? Si les positions de More et de Glanvill concernant la sorcellerie méritent l'attention, c'est plutôt parce qu'elles permettent de comprendre que la critique philosophique de l'enthousiasme peut, dans certains cas, être interprétée comme un facteur de crise religieuse.

\section{LES RAISONS STRATÉGIQUES \\ DU CHOIX D'UNE EXPLICATION MÉDICALE DE L'ENTHOUSIASME ET DE LA SORCELLERIE}

C'est dans Enthusiasmus Triumphatus (1656) que More met en œuvre le principe d'une explication médicale de l'enthousiasme ${ }^{7}$. Pour More, on peut

6. Stuart ClaRK dans Thinking with Demons. The Idea of Witchcraft in Early Modern Europe, Oxford, Oxford University Press, 1997, critique l'idée selon laquelle la révolution scientifique se serait traduite par un déclin de la croyance en la sorcellerie et met en évidence le rôle des savants et philosophes représentants de la nouvelle science expérimentale et membres de la Royal Society dans la défense de cette croyance. Sur ce point, cf. chap. XIX, pp. 294-311. Pour une vue synthétique de la littérature critique sur la sorcellerie, cf. Michael HunTER, "Witchcraft and the Decline of Belief », Review Essay, Eighteenth-Century Life 22.2, Johns Hopkins University Press, 1998, pp. 139-147.

7. Cf. l'article de David Leech. L'explication était déjà présente chez Meric CASAUBON un an auparavant dans A Treatise Concerning Enthusiasm (1655). 
réduire la prétention à l'inspiration à la forme hypochondriaque de la mélancolie. La tendance des enthousiastes à prendre le produit de leur imagination pour une réalité (à croire par exemple qu'ils sont en communication directe avec Dieu) s'explique par le processus d'adustion de l'humeur mélancolique qui s'accompagne de la production de vapeurs, lesquelles perturbent l'ensemble des facultés sensorielles de l'homme et produisent le délire de l'imagination ${ }^{8}$.

En adoptant ce principe explicatif, More et Glanvill n'entendent pas minimiser le danger politique et religieux que représente à leurs yeux l'enthousiasme. Ils ne cessent au contraire, tout au long de leurs écrits, de dénoncer le rigorisme des puritains ainsi que leur pratique du culte fondée sur la crainte du châtiment divin, pratique responsable selon eux de la défiance qui s'est exprimée pendant la guerre civile vis-à-vis des dogmes modérés de l'Église anglicane et de la prolifération des mouvements sectaires. Mais leur critique repose sur cette conviction que l'explication médicale du comportement des puritains et des adeptes des sectes dissidentes fera plus pour les discréditer et les empêcher de gagner des adeptes que l'explication démonologique qui, au contraire, tendra à accroître leur prestige aux yeux de la foule ignorante. Le choix de l'explication naturaliste est d'abord stratégique, comme l'explique More dans l'édition de ses œuvres complètes en $1662^{9}$.

Précisément, c'est le même objectif qu'affichent les adversaires de la croyance en la sorcellerie au moment où le débat renaît, avec des enjeux nouveaux, au milieu du XVII siècle. Hobbes fait en effet reposer la croyance en la sorcellerie, dans le chapitre II de la première partie du Léviathan (1651), sur l'ignorance des causes naturelles des phénomènes et sur l'incapacité à distinguer vision et sensation. Le pouvoir naturel de l'imagination, qui conduit les hommes à confondre le produit de leurs phantasmes avec la réalité, suffit à rendre compte des phénomènes de sorcellerie. Ce n'est cependant pas une raison pour ne pas les châtier dans la mesure où la croyance en la sorcellerie est responsable, comme celle en la réalité des dons revendiqués par les enthousiastes, de désordres politiques et religieux ${ }^{10}$.

Au même moment, l'ouvrage de Reginald Scot est réédité ${ }^{11}$. Enfin John Wagstaffe et John Webster donnent un écho important aux arguments de Hobbes

8. Voir par exemple le $\S 17$ de Enthusiasmus Triumphatus, or a Discourse of the Nature, Causes, Kinds, and Cure of Enthusiasme, written by Philophilus Parresiastes, and prefixed to Alazono Mastix his Observations and Reply, London, J. Flesher, 1656. The Cambridge Platonists, Henry More : Major Philosophical Works, ed. G. A. J. Rogers, Thoemmes Press, 1997 (nous renvoyons à cette édition des œuvres pour toutes les références données dans cet article, exception faite d'une référence à la réédition des œuvres supervisée par Henry More en 1662).

9. H. More, Enthusiasmus Triumphatus, in A Collection of Several Philosophical Writings of Dr. Henry More, London, Cambridge, W. Morden, 1662, § 67, p. 48.

10. Thomas Hobbes, Léviathan, I, 2, trad. F. Tricaud, Paris, Dalloz, 1999, p. 18.

11. Première édition en 1584 , l'ouvrage est réédité en 1651 puis en 1655. 
dans deux ouvrages entièrement consacrés à la question de la sorcellerie : le premier dans The Question of Witchcraft debated. Or a Discourse against their Opinion that affirm Witches en 1669, et le second dans The Displaying of Supposed Witchcrafts en $1677^{12}$. John Webster, médecin, disciple de Bacon et proche du milieu de la Royal Society, s'en prend, dans la Préface de son ouvrage, à Casaubon, More et Glanvill pour avoir épousé la cause de la sorcellerie, alors que Wier, Scot, Wagstaffe avaient réussi à mettre fin à cette croyance ${ }^{13}$.

\section{H. MORE ET J. GLANVILL,}

\section{DÉFENSEURS DE LA CROYANCE EN LA SORCELLERIE}

Accusés par John Webster d'être responsables d'un retour à l'obscurantisme en entretenant la croyance en la sorcellerie, Henry More et Joseph Glanvill rétorquent par le biais de différents écrits et sur un ton souvent polémique. More s'en prend en particulier à Hobbes et au Léviathan dans son Antidote against Atheism en 1653. Glanvill répond aux arguments de Reginald Scot dans A Blow of Modern Sadducism en 1665 et il répondra aussi à Webster au fil des textes qui vont aboutir à la publication posthume par Henry More de l'ensemble des textes de Glanvill sur la sorcellerie : Saducismus Triumphatus en $1681^{14}$.

Contre ceux qui entendent expliquer la sorcellerie par l'ignorance des causes naturelles, More et Glanvill se posent en défenseurs de cette croyance. L'explication qu'ils ont proposée pour rendre compte de l'enthousiasme ne peut être appliquée au cas des sorcières. More par exemple dénonce, dans la troisième partie de son Antidote contre l'athéisme, la manière dont on utilise les craintes superstitieuses qui habitent les mélancoliques comme prétexte pour rapporter les apparitions surnaturelles à une imagination perturbée. Quant à Glanvill, il s'étonne dès les premiers textes qu'il rédige sur cette question (Some Considerations about Witchcraft in a Letter to R. Hunt) que des hommes d'esprit ingénieux se soient entichés de cette lubie selon laquelle il n'y a ni sorcières ni apparitions ${ }^{15}$.

Leur plaidoyer passe, dans un premier temps, par un travail de compilation de récits extraordinaires témoignant de la réalité des phénomènes de sorcellerie.

12. The Displaying of Supposed Witchcraft. Wherein is affirmed that there are many sorts of Deceivers and Impostors, and Divers Persons under a Passive Delusion of Melancholy and Fancy. By John Webster, Practitioner in Physick. London, 1677. Selon T. H. JobE (« The Glanvill Webster Debate », Isis 72, 1981), l'ouvrage de Webster est achevé dès 1673.

13. J. Webster, op. cit., Preface, $§ 2$.

14. Voir par exemple sa réfutation des interprétations scripturaires de Webster dans A Proof of Apparitions, Spirits and Witches, from a choice Collection of moderne Relations (1681).

15. Some Considerations about Witchcraft..., in Collected Works of Joseph Glanvill (1661), facsimile ed., Hildesheim, New York, G. Olms, 1970, vol. IX, section I, p. 1. 
L'histoire du démon de Tedworth est, parmi tous les récits de sorcellerie compilés par More et Glanvill, l'un de ceux qui a le plus contribué à leur donner cette position de défenseurs de la croyance en la sorcellerie. L'affaire débute dans les années 1662-1663. Glanvill entend parler d'un démon joueur de tambour qui hanterait la demeure du comte de Mompesson. La présence du fantôme se manifeste par des coups frappés contre les murs et les portes. Glanvill se rend sur place pour mener sa propre enquête et il revient persuadé du caractère réellement surnaturel des phénomènes dont il a été le témoin. Dans son compte rendu, il prend soin de prévenir les objections des hobbesiens en précisant que la crainte n'est pas à l'origine de sa conviction :

Certains diront, je le sais, que mes amis et moi étions sous le coup de la crainte et avons ainsi imaginé des bruits et des visions qui n'étaient point. C'est là l'éternelle échappatoire. Mais en ce qui me concerne, je suis certain que pendant tout le temps où je me trouvais dans la chambre et dans la maison, je n'étais pas plus effrayé que je ne le suis pendant que je rédige ce récit ${ }^{16}$.

More de son côté, lorsqu'il rapporte cette histoire à son amie lady Conway, prend soin de préciser que des disciples de Hobbes ont été témoins de ces événements et qu'ils ont été convaincus de la réalité des événements surnaturels qui se sont produits dans la demeure du comte de Mompesson ${ }^{17}$. L'explication par la crainte, recevable dans le cas de l'enthousiasme, ne l'est pas lorsqu'il s'agit d'expliquer les phénomènes de sorcellerie. Les coups frappés par le démon de Tedworth n'auraient suscité aucune terreur panique chez ceux qui en sont témoins. Mais la question n'en demeure pas moins entière : pourquoi refuser le principe d'une explication naturaliste de la sorcellerie alors qu'il est admis pour l'enthousiasme?

\section{LA CRITIQUE PHILOSOPHIQUE \\ DE L'ENTHOUSIASME ET DE LA SORCELLERIE : FACTEUR DE CRISE RELIGIEUSE?}

On peut avancer deux raisons pour rendre compte de la position de More et de Glanvill, les deux étant liées à leur volonté de défendre la religion anglicane orthodoxe contre tout ce qui pourrait la menacer, de l'extérieur ou de l'intérieur.

16. Saducismus Triumphatus, or Full and Plain Evidence Concerning Witches and Apparitions (1689) by Joseph Glanvill. A facsimile reproduction with an introduction by Coleman O. Parson. Gainesville, Florida, Scholar's Facsimiles \& Reprints, 1966, Part. II, p. 103.

17. «Some Hobbians have been at Tedmouth and were convinced. » Henry More to Lady Conway, March 31, 1663. The Conway Letters, edited by Marjorie Hope Nicolson, revised edition by Sarah Hutton, Oxford, Clarendon Press, 1992, p. 216. 
De l'extérieur tout d'abord, il ne faut pas oublier que dans leur combat pour préserver la version modérée de l'anglicanisme, More et Glanvill n'ont pas un mais deux ennemis : l'athée et l'enthousiaste sont, comme More le déclare dès la préface de son Antidote, les deux plus grands ennemis de la religion établie ${ }^{18}$. Sont appelés athées ceux qui remettent en cause certains dogmes essentiels comme celui de la résurrection ou d'une vie future mais aussi ceux qui, comme Hobbes, nient l'existence d'êtres immatériels et expliquent toute chose, l'esprit y compris, par les lois de la nature. Or depuis les débuts du débat sur la sorcellerie, les partisans de l'explication médicale sont accusés de « sadducéisme », du nom des membres de cette secte juive qui rejetait l'idée de résurrection, le principe d'une vie future et d'une rétribution et croyait en l'existence d'un Dieu corporel. L'argument n'est pas nouveau. Il est déjà convoqué par Jacques $\mathrm{I}^{\mathrm{er}}$ dans sa Démonologie ${ }^{19}$, et on le rencontre aussi sous la plume de Thomas Browne dans sa Religio Medici (1635) ainsi que dans le Treatise of Specters (1658) de l'évêque Bramhall, adversaire de Hobbes. Mais dans le contexte de la publication du Léviathan, où toute explication naturaliste et matérialiste peut être perçue comme une attaque contre la religion, il acquiert un poids nouveau ${ }^{20}$.

Admettre le principe d'une explication naturelle de la sorcellerie c'est, aux yeux de More et de Glanvill, remettre en cause la croyance en l'existence d'un esprit diabolique, et donc la croyance aux esprits en général, ce qui conduit nécessairement à nier Dieu et l'immortalité de l'âme. Henry More parodie la formule célèbre utilisée par Jacques $\mathrm{I}^{\mathrm{er}}$ pour défendre l'idée d'une unité indissoluble entre l'Église et l'État (No Bishop, no King) et l'applique à ceux qui ne croient pas en la réalité des esprits démoniaques ou des sorcières en les présentant comme des athées en puissance :

Assurément cette affirmation - Pas d'évêque, pas de roi - était tout aussi vraie en politique que celle-ci - Pas d'Esprit, pas de Dieu (No Spirit, no God) - l'est dans le domaine de la métaphysique ${ }^{21}$.

18. «I know not what you mean said Mastix, your late laudable intentions, said I, have been as well against Enthusiasme as Atheisme. » H. More, An Antidote Against Atheism Or, An Appeal to the Naturall Faculties of the Minde of Man \& An Appendix to the late Antidote Against Atheism, The Cambridge Platonists, Henry More : Major Philosophical Works, ed. G. A. J. Rogers, Thoemmes Press, 1997, Preface, A4. Sur ce double combat contre l'enthousiasme sectaire et l'athéisme, voir Stuart Clark, op. cit., chap. XIX, p. 303.

19. Voir le titre du chapitre VII du livre II : «Those that denies the power of the Devil, denies the power of God, and are guiltie of the errour of the sadduces. » James I, Daemonologie, in forme of a Dialogue, divided into three bookes, Edinburgh, 1597, facsimile ed., Amsterdam, Da Capo Press, 1969, pp. 54-55.

20. Dans la lettre-préface de More à Saducismus Triumphatus, le nom de Webster est immédiatement associé aux noms des philosophes sur lesquels se concentrent les accusations d'athéisme au $\mathrm{XVII}^{\mathrm{e}}$ siècle : Hobbes et Spinoza.

21. H. More, Antidote Against Atheism, op. cit., Book III, chap. 16, p. 278. 
La position de ceux qui, comme Hobbes, nient les pouvoirs attribués aux sorcières est expliquée par le fait qu'ils ne veulent pas reconnaitre d'autre substance que la matière et comprise comme une remise en cause des phénomènes surnaturels sur lesquels se fondent la révélation et le christianisme. Comme le déclare Joseph Glanvill dans la préface de ses Considérations sur la sorcellerie (1661) :

L'athéisme commence par le sadducéisme, ceux qui n'osent pas dire franchement qu'il n'y a pas de Dieu se contentant (en guise de premier pas et de préambule courtois) de nier qu'il y ait des esprits ou des sorcières ${ }^{22}$.

De celui qui ne croit pas en l'existence des sorcières, on peut s'attendre à ce qu'il ne croie pas non plus en l'existence des anges, pas plus qu'en la résurrection des corps ou en l'immortalité de l'âme. Autrement dit, il est susceptible de remettre en cause tous les principes fondamentaux de la religion.

Plus généralement, c'est l'existence d'un principe spirituel irréductible à une cause matérielle et corporelle qui est en cause dans le débat sur la sorcellerie. Lorsque More introduit la troisième partie de son Antidote against Atheism (1651) dans laquelle il polémique avec Hobbes, il insiste sur cet enjeu inhérent au débat sur la sorcellerie. Il s'agit de prouver l'existence des esprits en présentant des cas :

[...] que l'on ne peut résoudre par le recours à des causes naturelles et que l'on ne peut imaginer être le résultat du hasard mais qui sont si miraculeux qu'ils impliquent nécessairement la présence d'une libre et subtile essence spirituelle distincte de la matière brute et du pouvoir ordinaire de la nature ${ }^{23}$.

Les exemples récents d'apparitions surnaturelles et de phénomènes de sorcellerie peuvent être considérés comme des bienfaits fournis par la providence pour lutter contre les philosophes qui offensent la religion en niant l'existence d'êtres immatériels ${ }^{24}$. La défense de la croyance en la sorcellerie sert de terrain de bataille pour réfuter les thèses de ceux qui réduisent tout à des principes matériels et qui sont de ce fait accusés d'athéisme.

22. Some Considerations, op. cit., Preface, p. 62. Voir aussi A Whip for the Droll, Fidler to the Atheist : Being Reflections on Drollery and Atheism, London, 1668, in Saducismus Triumphatus, ed. C. O. Parsons, 1966, sect. IV, p. 541 : «There is a latent atheism at the root of sadducean principle : for too many deny witches, because they believe there are no Spirits : and they are so perswaded because they own no being in the world, but matter, and the results of motion, and consequently, can acknowledge nothing of a God. »

23. Antidote Against Atheism, op. cit., Book III, chap. I, p. 161.

24. «I look upon it as a special piece of providence that there are ever and anon such fresh examples of Apparitions and Witchcraft [...]. » Dr H. M. his Letter with the Postscript to M. J.G. Saducismus Triumphatus, in Collected Works of Joseph Glanvill, Hildesheim-New York, Georg Olms Verlag, 1978, vol. IX, p. 16. 
Cette première raison ne résout pas pour autant le problème. On ne peut en effet manquer de se poser la question suivante : si les défenseurs de la religion anglicane que sont Henry More et Joseph Glanvill se sont aperçus de ce danger au sujet de la sorcellerie, pourquoi alors n'ont-ils pas adopté la même position concernant l'enthousiasme?

C'est en répondant à cette question que John Webster propose une hypothèse de lecture intéressante. Selon lui, les tensions et les difficultés rencontrées au fil de la critique de l'enthousiasme auraient conduit More et Glanvill à adopter une position purement défensive. L'explication naturaliste de l'enthousiasme par la corruption de l'humeur mélancolique les place en effet du côté des partisans d'une explication matérialiste des phénomènes surnaturels et donc des sadducéens ou des athées dont ils entendent pourtant se démarquer. Et Webster d'ajouter que c'est pour contrer cette accusation que Meric Casaubon aurait rédigé son traité prouvant les esprits, les sorcières et les opérations surnaturelles $^{25}$ (A Treatise proving Spirits, Witches, and Supernatural Operations, 1672).

Les positions de More et de Glanvill sur la sorcellerie résulteraient ainsi des tensions que suscite ce double combat. S'ils redoutent d'étendre aux sorcières le principe d'une explication naturaliste et médicale de leur comportement, ce n'est pas pour les mêmes raisons que Bodin ou que Jacques $\mathrm{I}^{\mathrm{er}}$. Le danger n'est pas que cette explication conduise à considérer les sorcières comme des victimes et à faire preuve de trop d'indulgence à leur égard, mais plutôt qu'elle porte directement atteinte aux intérêts de la religion établie.

Henry More s'est rendu compte assez tôt de ce risque. L'évolution de ses positions concernant l'enthousiasme en témoigne. Alors que le principe d'une explication naturaliste de l'enthousiasme est adopté clairement dans Enthusiasmus Triumphatus en 1656, en 1660, dans An Explanation of the Grand Mystery of Godliness, More semble abandonner l'explication médicale pour présenter ceux qui se donnent le titre de prophètes (Mahomet, Appolonius de Tyana, Henri Niclaes, fondateur de la secte de la Famille d'amour ${ }^{26}$ ) comme « des escrocs et des imposteurs, d'effrontés épicuriens », qui ont profité de leur tempérament enthousiaste pour tromper l'ensemble du monde chrétien ${ }^{27}$ et peuvent être considérés comme des agents du diable.

25. J. WeBSTER, The Displaying of Supposed Witchcraft, op. cit., chap. I, p. 8.

26. Secte fondée entre 1541 et 1590 par Henri Niclaes à Amsterdam. Le mouvement s'étend en Angleterre au $\mathrm{XVI}^{\mathrm{e}}$ siècle par l'intermédiaire de Christopher Vittells qui traduit en anglais les pamphlets de Niclaes. En affirmant qu'il est possible d'atteindre dès l'existence terrestre l'état de perfection qui était celui d'Adam avant la chute, cette secte remet en cause, aux yeux de ses adversaires, la doctrine d'une rétribution future et le dogme du péché originel.

27. H. MoRe, An Explanation of the Great Mystery of Godliness, "The Cambridge Platonists », Thoemmes Press, edited and introduced by G. A. J. Rogers, 1997, Book V, chap. VIII, § 7, p. 154. 
Ce que More redoute, c'est la possibilité d'étendre le principe d'une explication naturaliste à l'ensemble des phénomènes surnaturels qui fondent la révélation et la religion chrétienne. Wagstaffe par exemple, lorsqu'il explique la sorcellerie par la mélancolie, insiste sur le rôle joué par la crainte dans la genèse de la croyance en la réalité des pouvoirs surnaturels attribués aux sorcières ${ }^{28}$. Si la croyance en la sorcellerie s'explique par la crainte qui habite ceux dont l'imagination est perturbée par un tempérament mélancolique, alors pourquoi la croyance en Dieu ne s'expliquerait-elle pas elle aussi de cette manière ? Le fait que Wagstaffe ait ajouté en annexe de son plaidoyer en faveur de la croyance en la sorcellerie un dialogue de Lucien consacré à la critique de la superstition (Lover of Lyes) est clairement perçu comme une attaque directe contre la religion. L'argumentation de Wagstaffe s'accompagne en effet d'une charge anticléricale indirecte. Pour Wagstaffe, qui ne prend pas le risque de mentionner les prêtres de l'Église anglicane mais utilise la référence indirecte des religions païennes et des monarchies orientales, il est dans l'intérêt des hommes politiques comme des prêtres d'entretenir une pratique superstitieuse de la religion en maintenant le peuple dans la crainte des événements surnaturels ${ }^{29}$.

Les arguments utilisés par Wagstaffe pour dénoncer la stratégie qui sous-tend la croyance en la sorcellerie sont à peu près les mêmes que ceux que l'on trouvera sous la plume de Shaftesbury ou de Toland, lorsqu'il s'agira pour eux de dénoncer les impostures des prêtres. Or c'est justement contre cette tendance à transformer la critique de l'enthousiasme et de la superstition en un instrument de critique de la religion que More et Glanvill tentent de lutter. La critique rationnelle de l'enthousiasme doit, selon eux, être strictement limitée à la lutte contre le puritanisme et la prolifération sectaire. Elle ne doit pas servir à mettre en cause l'Église ni ses représentants. On comprend donc pourquoi les arguments utilisés par les adversaires de la croyance en la sorcellerie sont perçus par les défenseurs de l'anglicanisme modéré comme l'annonce d'une radicalisation de la crise religieuse amorcée avec le débat sur l'enthousiasme.

En dépit des efforts de More et Glanvill pour restreindre le principe d'une explication naturaliste au seul cas de l'enthousiasme et pour faire de la sorcellerie un rempart contre l'athéisme, leur démarche reste toutefois assez ambiguë $^{30}$. Tout en affirmant que les lois qui régissent le monde spirituel nous sont

28. Voir en particulier le chapitre vi de The Question of Witchcraft Debated: "How the Opinion of Witches came at first into the World", pp. 68-69.

29. J. Wagstaffe, The Question of Witchcraft Debated, op. cit., chap. I, pp. 1-2.

30. J. I. CoPE, Joseph Glanvill Anglican Apologist, St. Louis, Washington University Studies, 1956, chap. IV, pp. 96-97. 
inconnues, ils empruntent à la médecine et à la biologie de leur temps des concepts et des schémas d'explication susceptibles de rendre compte du comportement des sorcières. La notion médicale de contagion, à laquelle Webster avait recours pour discréditer le caractère surnaturel des pouvoirs attribués aux sorcières, sert par exemple chez Glanvill à affirmer la réalité du pouvoir de fascination que les sorcières exercent au moyen de leurs yeux (mauvais œil) ${ }^{31}$. La nature, écrit-il, agit par le biais de courants subtils ou par la diffusion de particules minuscules qui passent d'un corps à l'autre. On reconnaît ici l'hypothèse corpusculaire formulée par un membre célèbre de la Royal Society : Robert Boyle. Quant au pouvoir attribué aux sorcières de se déplacer dans les airs, il ne témoigne que de la possibilité d'une séparation de l'âme et du corps, possibilité dont les phénomènes d'apoplexie, d'épilepsie, d'extase, étudiés par les médecins, rendent compte.

Alors que pour ceux qui ne croient pas en la réalité des dons attribués aux enthousiastes ou aux sorcières, la référence à ces pathologies sert à démontrer que les pouvoirs qu'ils s'attribuent ne sont que les symptômes d'un dérèglement pathologique, elle est utilisée par Glanvill pour montrer que les pouvoirs attribués aux sorcières font tout autant partie de l'ordre naturel que les cas d'épilepsie ou que les phénomènes de contagion.

La tendance de Glanvill à revenir à des explications naturalistes parfois très proches de celles que l'on pourrait trouver sous la plume de John Webster par exemple ne peut donc pas être interprétée comme une incohérence pure et simple. Il faut au contraire tenter de l'expliquer (c'est la démarche interprétative proposée par Allison Coudert ${ }^{32}$ ) par la nature même de son projet. Glanvill, comme Webster, revendique l'héritage baconien. Dans la lettre qu'il adresse à un membre de la Royal Society (lord Brereton) en préface à A Blow of Modern Sadducism (1668), il propose de mettre en œuvre cette histoire naturelle des faits prodigieux susceptible, comme l'écrivait Bacon dans le livre II de The Advancement of Learning (1605), de nous aider à percer les secrets de la nature. Or il faut se rappeler que cette proposition de Bacon se fondait sur un constat sceptique : puisque nous ignorons dans quels cas et jusqu'à quel point les effets attribués à la superstition relèvent en réalité de causes naturelles, une enquête sérieuse et approfondie de ces phénomènes pourrait nous apporter des lumières utiles et nous aider à percer les secrets de la nature ${ }^{33}$.

31. Ibid., sect. V, p. 23.

32. Allison COUDERT, «More and Witchcraft », in Henry More (1614-1687). Tercentenary Studies, ed. Sarah Hutton, with a biography and bibliography by Robert Crocker, Dordrecht, Boston, London, Kluwer Academic Publishers, Archives internationales d'histoire des idées, 127, 1990, pp. 115-136.

33. F. BACON, The Advancement of Learning, Works, ed. J. Spedding and R. L. Ellis, London, 1870-1872, 4 : 296. 
L'héritage baconien est interprété par les partisans de la croyance en la sorcellerie et par ses détracteurs selon deux directions très différentes. D'une part, en ce qui concerne la finalité d'une enquête relative aux phénomènes surnaturels (sorcellerie, divination, charmes...), on voit bien que pour Webster, partir à la recherche des causes naturelles de ces phénomènes, c'est montrer que ce que les hommes attribuent par ignorance au pouvoir du diable relève en réalité des lois de la nature. Au contraire, pour Glanvill, cette enquête doit permettre de faire passer la sorcellerie du statut de superstition à celui de science expérimentale, et en même temps de combattre le sadducéisme. D'autre part, le doute dans lequel les hommes se trouvent, selon Bacon, au sujet des phénomènes surnaturels, est interprété par les deux camps de manière très différente. L'ignorance des véritables causes des phénomènes explique, selon Webster, que l'on attribue aux sorcières des pouvoirs qui pourraient s'expliquer naturellement si nous progressions dans notre connaissance du monde matériel ${ }^{34}$. De son côté, Glanvill se fonde lui aussi sur une argumentation sceptique pour défendre la réalité des dons extraordinaires attribués aux sorcières. Nous ne devons pas espérer parvenir à un degré de certitude plus grand dans notre investigation des phénomènes surnaturels que celui auquel nous pouvons prétendre dans l'investigation des phénomènes naturels. Or, lorsque nous étudions la nature, explique Glanvill en s'inspirant ici d'un argument probabiliste, nous ne sommes pas en mesure d'éclaircir la nature même des causes des phénomènes ${ }^{35}$. Pourtant, pas plus que l'ignorance de la cause permettant d'expliquer le processus de formation du fœtus dans l'utérus ne nous fait douter de la réalité de ce phénomène naturel, l'ignorance dans laquelle nous nous trouvons de la cause naturelle permettant d'expliquer comment les sorcières se déplacent dans les airs ou se métamorphosent en animaux ne doit nous empêcher de croire en la réalité de ces phénomènes surnaturels ${ }^{36}$. L'ignorance des causes naturelles qui servait, dans le texte de Webster, à refuser la croyance en la sorcellerie est utilisée, par Glanvill, pour la fonder. De même, le caractère invraisemblable des faits rapportés par ceux qui ont été témoins des phénomènes de sorcellerie constitue, aux yeux de Glanvill, un argument en faveur de leur caractère authentique. Plus un récit de sorcellerie apparaît en contradiction avec ce que la raison commune

34. « [...] those many elucubrations and continued discoveries of those learned and indefatigable persons that are of the Royal Society [...] do plainly evince that hitherto we have been ignorant of almost all the true causes of things, and therefore through blindness have usually attributed those things to the operation of cacodemons that were truly wrought by nature and thereby not smally augmented and advanced this gross and absurd opinion of the power of witches. » J. WEBSTER, op. cit., pp. 267-268.

35. «For in resolving natural phaenomena, we can only assign the probable causes, sheing how things may be, not presuming how they are. » J. Glanvill, Some Considerations, op. cit., p. 13.

36. Ibid., p. 15. 
peut nous porter à admettre comme vrai, et moins il paraît probable de supposer que les différents témoins qui en rendent compte aient pris le risque de passer pour fous en inventant de toutes pièces de telles histoires. Comment en effet, demande Glanvill, l'imagination, qui est la chose la plus protéiforme au monde, pourrait-elle être à l'origine des mêmes lubies en tous temps et en tous lieux ${ }^{37}$ ?

On ne peut donc se contenter d'interpréter la défense de la réalité des pouvoirs attribués aux sorcières comme un reste de superstition chez les partisans d'un examen rationnel des pratiques religieuses. Contre ceux qui pensent que tout peut s'expliquer dans l'univers à partir de principes matériels, More et Glanvill entendent montrer la nécessité d'un principe spirituel, seul capable de rendre intelligibles les phénomènes naturels. Il s'agit aussi pour eux de montrer que la science nouvelle mise en œuvre par les membres de la Royal Society, l'appel à l'expérience et l'investigation des causes naturelles des phénomènes, loin de porter préjudice aux intérêts de la religion, doivent contribuer à la conforter en luttant contre ceux qui la menacent, qu'il s'agisse des athées ou des enthousiastes $^{38}$. Tout comme l'explication rationnelle et naturaliste de l'enthousiasme doit servir à écarter la menace du radicalisme religieux des puritains, la science naturelle et expérimentale de la sorcellerie doit servir à écarter la menace du matérialisme en démontrant la réalité des phénomènes surnaturels et du monde des esprits. Il s'agit bien, dans les deux cas, de défendre les intérêts de la religion en se fondant sur les progrès de la science expérimentale ${ }^{39}$. Paradoxalement et certainement contre sa volonté, il n'en demeure pas moins qu'en proposant de fonder une histoire naturelle des faits surnaturels, Glanvill a ouvert la voie aux déistes qui, comme Shaftesbury ou John Trenchard, proposeront de généraliser cette démarche à l'ensemble des phénomènes surnaturels sur lesquels se fondent les religions révélées.

\section{CRignon-De Oliveira} Université Paris VIII

37. Ibid., pp. 10-11.

38. Voir le titre de l'ouvrage publié par GlanviLl en 1671 : Philosophia Pia : or a Discourse of the Religious Temper and Tendencies of the Experimental Philosophy.

39. Rappelons que Boyle publie en 1675 un texte intitulé Some Physico-theological Considerations about the Possibility of the Resurrection. 